\title{
Las concepciones modelísticas y la concepción estructuralista de las teorías*
}

The Modelist Views and the Structuralist View of Theories

C. Ulises Moulines ${ }^{\dagger \ddagger}$

\begin{abstract}
Resumen
Se exponen y analizan críticamente las concepciones epistemológicas y metodológicas que han dominado la filosofía de la ciencia durante el último tercio del siglo XX y comienzos del siglo XXI. Todas ellas revelan ciertas semejanzas entre sí, que permiten agruparlas bajo una misma familia de concepciones que podemos describir como "modelísticas" o "semanticistas", pues en ellas la noción semántica de "modelo" desempeña un papel central -si bien también hay divergencias importantes entre ellas-. Las concepciones modelísticas consideradas aquí son: la axiomática conjuntista de la Escuela de Stanford, el representacionalismo de Patrick Suppes y sus colaboradores, el representacionalismo de Günther Ludwig, los enfoques emparentados de Bas van Fraassen, Frederick Suppe y Ronald Giere, así como el estructuralismo metateórico fundado por Joseph D. Sneed y Wolfgang Stegmüller.
\end{abstract}

Palabras clave: modelo - teoría científica - experiencia - representación

\begin{abstract}
In this article, I describe and critically analyze those epistemological and methodological views that have been preponderant in the philosophy of science of the last third of the 20th century as well as of the beginning of the 21th century. They all reveal some similarities that allow grouping them under just one family of approaches, which may be labelled "modelist" or "semantic" since the semantic notion of a "model" plays a central role in them - though there also are important divergences between them. The modelist views here considered are: the set-theoretical axiomatics of the Stanford School, the representationalism of Patrick Suppes and his collaborators, Günther Ludwig's representationalism, the related approaches of Bas van Fraassen, Frederick Suppe, and Ronald Giere, as well as the metatheoretical structuralism founded by Joseph D. Sneed and Wolfgang Stegmüller.
\end{abstract}

Keywords: model - scientific theory - experience - representation

* Recibido: 15 de septiembre de 2012. Aceptado con revisiones: 11 de noviembre de 2012.

${ }^{\dagger}$ Munich Center for Mathematical Philosophy, University of Munich, Alemania. Para contactar al autor, por favor, escribir a: moulines@lrz.uni-muenchen.de.

* Este trabajo reproduce, con algunas leves modificaciones, parte del capítulo 6 del libro Moulines, C. U., El desarrollo moderno de la filosofía de la ciencia (1890-2000), México: UNAM-IIF, 2011 -el cual, a su vez, es una versión castellana, debida a Xavier de Donato, de Moulines, C. U., Die Entwicklung der modernen Wissenschatfstheorie (1890-2000) - Eine historische Einführung, Hamburg: Lit Verlag, 2008-.

Metatheoria 5(2)(2015): 7-29. ISSN 1853-2322. eISSN 1853-2330.

(C) Editorial de la Universidad Nacional de Tres de Febrero. Publicado en la República Argentina. 


\section{Introducción}

Es difícil encontrar una característica común a los diversos autores y corrientes que han desempeñado, o desempeñan todavía, un papel significativo durante la última fase de la historia de nuestra disciplina, que ocupa aproximadamente las últimas tres décadas del siglo XX. Falta, además, la perspectiva que da el transcurso del tiempo. Sin embargo, podemos observar un "aire de familia" en la mayor parte de representantes importantes de la filosofía de la ciencia durante este período. Establecer algunos rasgos comunes -aun cuando hayamos de describirlos de un modo un tanto vago, pues admiten realizaciones diferentes en cada enfoque- nos ayudará a medir el camino recorrido por nuestra disciplina desde 1970.

Se constata, en primer lugar, una profunda desconfianza (si no una franca aversión) hacia una metodología casi exclusivamente sintáctico-formal en el análisis de los conceptos y las reconstrucciones de las teorías científicas emprendidas por muchos de los autores más influyentes de la fase "clásica" (Carnap, Hempel, Nagel, Braithwaite e incluso Popper y sus discípulos). He ahí un punto común entre los autores de la fase que estamos considerando y los "historicistas" (Kuhn, Feyerabend, Lakatos y Laudan), pero su rechazo del "sintacticismo" obedece a motivaciones completamente diferentes: no es que den la primacía a una metodología historiográfica (ligada a la idea muy extendida según la cual "la historia de las ideas no se deja analizar formalmente"), ni que recusen los métodos más o menos formales de análisis. Al contrario, un cierto número de autores de los tres últimos decenios, consideran que los problemas que enfrentaron los análisis clásicos de las teorías científicas provienen de la aplicación de instrumentos formales demasiado elementales (básicamente la lógica de predicados de primer orden) y que más bien deben utilizarse instrumentos lógico-matemáticos "fuertes" (teoría de conjuntos, topología, teoría de modelos, lógicas modales e incluso nociones no triviales de la informática) para dar cuenta de aquello que es esencial en las teorías, sobre todo en las disciplinas fuertemente matematizadas como la física y una gran parte de la química, de la biología y de las ciencias sociales.

La noción puramente sintáctica o cuasi-sintáctica de teoría científica, en tanto que conjunto de axiomas con sus consecuencias lógicas (noción característica de la fase clásica), no se considera adecuada a la complejidad de las estructuras conceptuales y metodológicas de las ciencias empíricas. La noción de teoría es reinterpretada en un marco semántico o semántico-pragmático, o incluso completamente reemplazada por la noción de modelo, cuya definición varía, no obstante, según los autores. Se puede, pues, recurrir al neologismo "modelístico" para intentar caracterizar los rasgos comunes a las concepciones que aquí nos interesan. En ciertos autores, la noción de modelo está definida de manera formal (con ayuda de la teoría de conjuntos, por ejemplo), en otros es usada de manera más informal; pero, en todos los casos, se considera que son los "modelos", en tanto que representaciones (parciales e idealizadas) de "pequeñas partes" de la realidad (o de la experiencia humana) lo que constituye la "substancia" del conocimiento científico.

Desde un punto de vista epistemológico general, la mayoría de los autores de este período es abierta o implícitamente antirrealista, si entendemos por "realismo científico" la creencia en el presupuesto de que el objetivo de las teorías científicas es el de reflejar, de una manera más o menos aproximada, la realidad de la naturaleza "tal y como es". Una cierta dosis de "instrumentalismo" en sentido amplio está presente en muchos de estos autores: los modelos propuestos por las ciencias son instrumentos que nos permiten orientarnos en un campo de la experiencia humana demasiado complejo para ser completa y fielmente reflejado por una sola teoría.

De manera general, los factores pragmáticos que intervienen en la constitución de la estructura y el funcionamiento de las teorías científicas son tomados en cuenta bastante seriamente, y en algunos autores podemos encontrar intentos de sistematización de la dimensión pragmática de la ciencia, a veces incluso formalizados. No obstante, esta "pragmatización” del análisis de la ciencia (es decir, la 
consideración de factores ligados a los intereses de la comunidad científica que construye sus modelos de representación de la experiencia) no equivale a una "sociologización" pura y simple de la noción de teoría o del método científico -como fue el caso de muchas interpretaciones historicistas-. Y menos aún se trata de caer en la trampa del relativismo socio-epistémico.

Los estudios de caso de teorías científicas concretas se vuelven muy importantes: sin ser considerados como valiosos en sí mismos, son utilizados como "tests" para poner a prueba o para ilustrar una visión más general de la ciencia, o al menos de una disciplina particular (como la física o la biología). Al mismo tiempo, se constata una tendencia (metodológica e incluso epistemológica y ontológica) al pluralismo: la idea de que no existiría más que una manera esencial de "hacer ciencia", o un único método formal para aplicar las directrices del espíritu científico, se cuestiona de raíz.

Sin embargo, los diversos representantes de una concepción "modelística" de la disciplina no rechazan de plano las contribuciones de los autores del positivismo lógico y de la filosofía clásica de la ciencia. Muchos conceptos, temas y problemas de las fases precedentes -tales como la relación entre la teoría y la experiencia, la noción de explicación científica, el papel de las formas de inferencia no deductiva, el concepto de ley científica, la noción de reducción entre teorías, etc.- vuelven a entrar en escena, aunque bajo una interpretación diferente.

Estudiemos ahora algunos de los enfoques más característicos o los más conocidos de la "nebulosa modelística". Si bien la lista no es exhaustiva, sin embargo es significativa. Examinaremos estos enfoques en orden cronológico, a partir del momento en que tomaron por primera vez una forma suficientemente reconocible; no olvidemos sin embargo que en el momento en que escribimos estas líneas, todos estos enfoques (con excepción quizá del primero) siguen desarrollándose, de tal suerte que es difícil dar una imagen definitiva de ellos.

\section{La concepción conjuntista de la escuela de Stanford}

Nos permitimos introducir aquí un neologismo poco atractivo ("conjuntismo") para designar los trabajos de un grupo de lógicos y filósofos de la ciencia, activos sobre todo en la Universidad de Stanford (Estados Unidos), que han aplicado sistemáticamente los conceptos y los principios de la teoría de conjuntos a la reconstrucción y al análisis de las teorías de las ciencias empíricas, principalmente de la física clásica (incluida la teoría especial de la relatividad). El término "concepción conjuntista" se propone, pues, como la traducción más satisfactoria de la expresión anglosajona settheoretical view.

Desde el punto de vista estrictamente cronológico, los principales trabajos de esta escuela pertenecen aún a la fase "clásica" de la filosofía de la ciencia, pues la mayor parte fueron publicados en los años cincuenta y sesenta. Sin embargo, el desarrollo del método "conjuntista" y su aplicación a un gran número de estudios de caso se llevó a cabo de una forma casi totalmente independiente de las discusiones características de la fase clásica, y el método reconstructivo de los miembros de la escuela de Stanford tuvo una gran influencia, a menudo explícitamente reconocida, sobre los autores de esta última fase de la filosofía de la ciencia.

Patrick Suppes (Estados Unidos, 1922-2014) es, sin duda, el representante más eminente de esta escuela. En colaboración con el lógico J.C.C. McKinsey y otros investigadores, tuvo la idea de axiomatizar las teorías empíricas de una manera mucho más simple y "transparente" conceptualmente que las tentativas precedentes, lo que no sólo le permitió comprender más fácilmente la estructura interna "esencial" de la teoría así reconstruida, sino también examinar adecuadamente sus eventuales relaciones con otras teorías. El método reconstructivo propuesto por Suppes y sus colaboradores es conocido bajo el nombre de "axiomatización por definición de un predicado conjuntista" (axiomatization by definition of a set-theoretical predicate). Estrictamente, no inventaron este método: se inspiraron en ciertas ideas del gran lógico Alfred Tarski (McKinsey fue discípulo suyo), que ya habían sido puestas en práctica en las teorías matemáticas, y en los trabajos de sistematización de las matemáticas emprendidos por el grupo de Bourbaki durante los años cuarentas y cincuentas. Sin 
embargo, aparte de algunas diferencias un tanto técnicas en la manera de desarrollar el método, Suppes y sus colaboradores aportaron una gran innovación a la filosofía de la ciencia, al mostrar de manera convincente que uno puede reconstruir de este modo prácticamente cualquier teoría científica empírica, dándole de esta forma su criterio de identidad con una gran precisión, sin que la tarea se vea sobrecargada por el aparato lógico considerado anteriormente como necesario para la reconstrucción de teorías. La teoría de conjuntos utilizada para aplicar el método "stanfordiano" consiste únicamente en lo que habitualmente se llama la teoría "informal" o "intuitiva" de conjuntos, la cual no está a su vez axiomatizada formalmente, pero que representa el aparato matemático más próximo desde un punto de vista conceptual de lo que es requerido en la práctica científica (y en el análisis epistemológico). De hecho, Suppes es probablemente el primer filósofo de la ciencia en darse cuenta de que la lógica de predicados de primer orden (la herramienta de análisis favorita de los positivistas lógicos y de los filósofos clásicos de la ciencia) no es un buen instrumento formal de análisis y de reconstrucción de la ciencia; al no servir más que para el análisis de cuestiones muy elementales, este instrumento lógico es a la vez "demasiado burdo" y "demasiado simple". El método conjuntista tiene otra ventaja: permite determinar directamente y sin recurrir a la construcción de un sistema de semántica formal, cuáles son los modelos de una teoría dada, es decir, las entidades conceptuales que supuestamente representan las diferentes partes del mundo de la experiencia; los modelos de una teoría son simplemente las entidades que satisfacen lo que se llama un predicado conjuntista. Además, esta determinación de la noción de modelo nos permite comprender de una sola vez que una teoría cualquiera producirá en general un número indeterminado de modelos realmente diferentes para representar la realidad -y no uno solo, como numerosos filósofos suponían a menudo-. En fin, como enseguida veremos, el método permite detectar directamente la forma lógica de los "compromisos ontológicos" de la teoría así reconstruida -lo que, seguramente, también es posible en el método clásico axiomático-formal, pero de una manera mucho menos directa y transparente-.

¿En qué consiste exactamente el método de "axiomatización por definición de un predicado conjuntista"? No entraremos aquí en los detalles técnicos, pero un ejemplo, ciertamente muy simplificado, aclarará nuestra exposición. Supongamos que en un manual de sociología o de psicología encontramos, por ejemplo, una "teoría" que nos habla de relaciones familiares y nos explica que las familias son entidades complejas constituidas por un padre y una madre, ligados entre sí por el matrimonio, y al menos un hijo engendrado por ellos. ¿Cuál es exactamente la identidad de esta teoría (y sus modelos)? La respuesta está dada por la definición del siguiente predicado conjuntista:

$x$ es una familia si y sólo si $x$ es una tupla (en el sentido de la teoría de conjuntos) constituida por un conjunto básico de al menos tres elementos y por dos relaciones diádicas (en el sentido de la teoría de conjuntos), llamadas "matrimonio" y "engendrar", que satisfacen ciertas propiedades que se pueden formular con ayuda de la teoría de conjuntos (por ejemplo, la relación "matrimonio" es irreflexiva y simétrica, mientras que la relación "engendrar" es asimétrica). Además, postulamos la "ley" de que, en toda familia, hay al menos un elemento que ha sido engendrado por otros dos elementos. ${ }^{1}$

\footnotetext{
${ }^{1}$ Para los lectores que tienen alguna familiaridad con las fórmulas lógico-matemáticas, he aquí, a modo de ilustración, la formalización conjuntista de esta mini-teoría de la familia. (Usamos las abreviaturas: "F" para "... es una familia"; "P" para un conjunto de personas, " $M$ " para la relación de matrimonio y "E" para la relación de engendrar. Para abreviar, escribiremos, para toda relación diádica $R$, "xRy" en lugar de la formulación " $\langle x, y\rangle \in R$ ”).

$F(X)$ si y sólo si $\exists P, M, E$ :

(0) $\quad X=\langle P, M, E\rangle$

(1) $P \neq \varnothing \wedge\|P\|>2$

(2) $\mathrm{M} \subseteq P \times P \wedge \forall x \forall y(\neg x \mathrm{M} x \wedge(x \mathrm{M} y \rightarrow y \mathrm{M} x))$

(3) $E \subseteq P \times P \wedge \forall x \forall y(x E y \rightarrow \neg y E x)$

(4) $\exists x, y, z \in P(x M y \wedge x E z \wedge y E z)$.
}

Señalemos que, dejando aparte los símbolos $P, M$ y $E$, que son conceptos primitivos específicos de nuestra teoría, todos los otros símbolos que aparecen en esta axiomatización conjuntista son nociones estándares de la lógica y de la teoría de conjuntos. La "ley empírica", según la cual en toda familia hay alguien que ha sido engendrado por otras dos personas, es expresada por la condición o axioma (4). De las 
Cada entidad concebida como una instancia de la variable $x$ en esta definición es pues una estructura consistente en un conjunto finito de base (las personas en cuestión) y dos relaciones diádicas ("matrimonio" y "engendrar") que deben satisfacer algunas condiciones expresables en teoría de conjuntos. Ahora bien, una estructura tal es precisamente un modelo de la teoría. ${ }^{2}$

La escuela de Stanford muestra que no existe, en principio, ningún obstáculo formal para identificar, con la precisión necesaria, todas las teorías científicas, aun las teorías más complejas de la física, así como sus modelos, por medio del método descrito. Además, una vez que la teoría y sus modelos han sido identificados, se puede hacer de manera bastante fácil un análisis formal de sus particularidades: por ejemplo, qué condiciones deben ser tomadas como axiomas y cuáles como teoremas, cuáles son los conceptos básicos (no definibles) requeridos, cuál puede ser la relación entre esta teoría y otra semejante, etc.

Más que hacer declaraciones programáticas generales o comprometerse con largas argumentaciones filosóficas, Suppes y sus colaboradores juzgaron más convincente "pasar a la acción" y reconstruir tantas teorías científicas "serias" como fuera posible. Su primera reconstrucción fue un hito en tanto que "paradigma" de la aplicación del método: la axiomatización conjuntista de la mecánica clásica de partículas, en un artículo de 1953 titulado precisamente "Axiomatic Foundations of Classical Particle Mechanics” [Fundamentos axiomáticos de la mecánica clásica de partículas]. A partir de entonces, un gran número de teorías de la física, la psicología y la economía fueron axiomatizadas por Suppes mismo o por otros. Todas estas reconstrucciones fueron publicadas de manera más o menos dispersa en muchas revistas y compilaciones. Suppes dio algunos ejemplos en la última parte de su libro Introduction to Logic (1957) [Introducción a la lógica], cuyo título es un poco equívoco pues no se trata meramente de un manual de lógica, sino de una exposición del método conjuntista de reconstrucción de las teoría científicas. En 1970, reunió aún más ejemplos, sobre la base de una exposición general del método y sus ventajas, en la obra Set-Theoretical Structures in Science [Estructuras conjuntistas en la ciencia], que, a pesar de la influencia que ejerció en los filósofos "reconstructores" de teorías científicas, curiosamente circuló durante muchos años sólo en forma de manuscrito. Una parte importante de los trabajos de Suppes sobre el método conjuntista fue recogida más tarde en Models and Methods in the Philosophy of Science (1993) [Modelos y métodos en filosofía de la ciencia].

A pesar de los éxitos innegables de su programa, Suppes no parece en condiciones de resolver de manera satisfactoria ciertas cuestiones epistemológicas y metodológicas fundamentales sobre la naturaleza de las teorías empíricas. Señalemos un punto crítico. Si identificamos los modelos de una teoría por medio de un predicado conjuntista, parece claro que si la teoría no es contradictoria o empíricamente inaceptable, encontraremos que, en principio, son aplicables un elevado número de sus modelos, no sólo uno. Este aspecto de la concepción suppesiana es completamente convincente a nivel intuitivo, pues siempre se constata que cuando los científicos disponen de una buena teoría, en general ésta no sólo es aplicable a una parte de la realidad, sino a varias. (Este hecho podría ser descrito como el "plurimodelismo" de las teorías empíricas.) Sin embargo, si uno identifica una teoría con todos los modelos determinados por el predicado conjuntista que la caracteriza, esta manera de concebir la identidad de las teorías científicas tropieza con un problema epistemológico grave: para decirlo brevemente, el número de estructuras que son modelos de una teoría definida por el método de Suppes, no sólo suele ser muy grande, sino demasiado grande. En efecto, siempre podemos encontrar un gran número de estructuras que satisfagan las condiciones conjuntistas del predicado y que, por tanto, sean formalmente modelos de la teoría. De hecho, para una teoría cualquiera que no sea completamente trivial, habrá siempre un número infinito de modelos efectivamente diferentes. (Esto es un resultado de un teorema bastante elemental de la teoría formal de modelos.) Para volver a nuestro ejemplo de la "teoría de la familia", sean cuales sean las condiciones más o menos restrictivas que

condiciones (2), (3) y (4) tomadas conjuntamente, se sigue el "teorema” según el cual las tres personas involucradas en (4) son siempre diferentes entre ellas. El “compromiso ontológico” de esta "teoría” está determinado únicamente por la presencia del conjunto $P$.

2 Para volver a la formalización de la nota precedente, cada estructura $\langle P, M, E\rangle$ que satisfaga las condiciones $(0)-(4)$ es, pues, por definición, un modelo de la teoría de la familia. Se puede probar que esta noción de modelo equivale, salvo en algunos casos particulares, a la noción de modelo de la semántica formal introducida por vez primera por Tarski y que se ha vuelto habitual en los manuales de lógica. 
impongamos a las relaciones que entran bajo la caracterización del predicado " $x$ es una familia”, este predicado será satisfecho no sólo por objetos que consideraríamos intuitivamente como siendo una "familia real", sino también por otros objetos que no tienen absolutamente nada que ver con ellas, pero que satisfacen las mismas condiciones conjuntistas (por ejemplo, dominios finitos que consisten en un número superior a dos elementos, y sobre los cuales es posible definir una relación irreflexiva y simétrica y una relación asimétrica). Se puede tratar incluso de estructuras matemáticas, cuyos conjuntos base contienen simplemente números como elementos, y que nada tienen que ver con el mundo empírico... ni a fortiori con el mundo de las familias.

Suppes mismo es más o menos consciente de este problema de interpretación del contenido de las teorías empíricas, pero no lo considera muy importante. Para él, este resultado muestra que, aun cuando en la construcción de una teoría empírica estemos generalmente guiados por intuiciones más o menos vagas concernientes al dominio de experiencia al cual debe ser aplicada, una vez la teoría está bien (re)construida, constataremos siempre que tiene muchas aplicaciones que no habíamos sospechado inicialmente, e incluso aplicaciones en dominios no empíricos (puramente matemáticos, por ejemplo). De hecho, Suppes no cree en la distinción esencial entre ciencias empíricas y ciencias matemáticas.

Para un filósofo de la ciencia "tradicional", esta respuesta no es satisfactoria, puesto que una de las grandes cuestiones de la filosofía de la ciencia desde sus inicios (sea cual sea la concepción particular defendida) es justamente distinguir las teorías empíricas de las no empíricas, y de hacer una distinción, en las ciencias empíricas, entre una disciplina y otra. Uno de los miembros más representativos de la escuela de Stanford, Ernest W. Adams (Estados Unidos), tematizó este problema y propuso otra solución. ${ }^{3}$ Según él (y en esto lo han seguido la mayoría de los autores de la nebulosa modelística), es insuficiente caracterizar una teoría científica empírica mediante una clase $M$ de modelos definida por un predicado conjuntista. Esta clase representa solamente una parte (si bien esencial) de su identidad. Para tener una plena comprensión de la teoría de que se trata, hay que añadir a la clase $M$ una clase I de aplicaciones o interpretaciones intencionales (intended interpretations), que nos indican el dominio de la experiencia al cual estos modelos deben ser aplicados. Formalmente, los elementos de I son estructuras del mismo tipo (es decir, concebidos de la misma manera) que los elementos de $\mathrm{M}$, sin que se pueda saber a priori si son verdaderamente modelos actuales de la teoría, es decir, estructuras que satisfacen las condiciones substanciales del predicado conjuntista. La "esperanza" del científico consiste justamente en creer que a la larga se podrá mostrar que $I$ es verdaderamente un subconjunto de $M$, es decir, que todas las aplicaciones intencionales satisfacen efectivamente todas las condiciones substanciales necesarias para ser modelos de esta teoría. Esta esperanza constituye lo que Adams llamó la "afirmación empírica" (empirical claim) de una teoría.

La noción de aplicación intencional, aun siendo parcialmente formalizable en los términos de la teoría de conjuntos, excluye a priori toda suerte de estructuras más o menos "grotescas" (desde un punto de vista intuitivo) o incluso puramente matemáticas que, "por azar", satisfagan las condiciones del predicado conjuntista. Ahora bien, de esta forma lo que estamos introduciendo en la noción de teoría empírica es un elemento irreductiblemente pragmático: las aplicaciones (o interpretaciones) intencionales lo son para alguien; y este "alguien", en el estado actual de las cosas, no es otro que la comunidad científica que, en un momento dado, construye o aplica la teoría en cuestión. La noción de teoría empírica depende así esencialmente de la noción de comunidad científica, la cual es claramente pragmática. Es ella la que selecciona las estructuras que vale la pena intentar comprobar si son efectivamente modelos de la teoría.

Hay, sin embargo, un problema lógico-matemático en esta concepción, sutil pero profundo, al cual Adams no da una respuesta convincente: en la determinación de la clase $I$, no se trata sólo de tener en cuenta los deseos o intenciones de los científicos, sino también de identificar cada uno de los

\footnotetext{
${ }^{3}$ Ver la Introducción a su artículo de 1959: "The Foundations of Rigid Body Mechanics and the Derivation of its Laws from those of Particle Mechanics" [Los fundamentos de la mecánica del sólido rígido y la derivación de sus leyes a partir de las de la mecánica de partículas].
} 
elementos de I, dado que es la única manera factible de determinar verdaderamente aquello de lo que queremos hablar en nuestra teoría. Ahora bien, en la concepción de Adams estamos constreñidos a presuponer, para la descripción de cada elemento de $I$, las nociones y las condiciones satisfechas por el predicado conjuntista característico de la teoría. De ahí que la "afirmación empírica" de la teoría, es decir, la proposición "I es un subconjunto de M", pase a ser irremediablemente auto-justificativa -lo que parece contradecir nuestras intuiciones de lo que significa "verificar una teoría empírica"-. En términos un tanto metafóricos, cada teoría se tornaría así un mundo cerrado en sí mismo... Volveremos sobre este punto cuando tratemos de la concepción estructuralista.

Otra manera de intentar resolver el problema de la distinción epistemológica entre ciencias empíricas y ciencias matemáticas consiste en efectuar un análisis profundo del sentido en el que se puede decir que un modelo cualquiera de una teoría es una estructura que pretende representar algunos aspectos de la realidad, y muy especialmente los aspectos que son dados por nuestra experiencia más o menos inmediata (particularmente lo que se llama "experimento de laboratorio"). Suppes mismo, sus discípulos, pero también otros investigadores que trabajaban independientemente, continuaron esta vía de maneras diversas.

\section{El representacionalismo}

La noción de modelo, tan central para la mayor parte de los autores que examinamos en esta presentación, está naturalmente ligada a la de representación: se supone que los científicos construyen modelos que representan más o menos bien partes de la realidad. Pero la noción misma de representación está lejos de ser clara a priori, en particular en un contexto científico. ¿Qué tipo de relación se designa con este término? En la estela de los trabajos de Suppes y su escuela, esta cuestión adquiere máxima importancia. El objetivo principal de la ciencia sería proporcionar representaciones más o menos adecuadas de la experiencia, y la tarea del filósofo de la ciencia es justamente determinar la naturaleza de esta relación de representación.

La representación de la que aquí se trata no se parece a la acción de un espejo que refleja un objeto tal y como es. Los objetos que deben ser representados por modelos de teorías científicas son siempre, en cierto modo, objetos estructurados conceptualmente. Por ejemplo, el objeto llamado "familia Pérez", que debe ser representado por un modelo de la "mini-teoría" de las relaciones de parentesco descrita más arriba, no es un objeto de la "experiencia pura" del sociólogo o del psicólogo, sino un objeto concebido estructuralmente de una cierta manera, en la que ciertas propiedades y relaciones son esenciales y otras, que uno podría tener en cuenta (como por ejemplo el color del cabello de los miembros de la familia), no lo son. En suma, los objetos estudiados por las teorías científicas son dominios simplificados y estructurados de una cierta manera. En la representación científica se trata de proceder de tal modo que los modelos de la teoría, que son ellos mismos estructuras, representen lo mejor posible estos dominios estructurados dados empíricamente. Sin embargo, no se puede presuponer que, en la representación, hay un acuerdo estructural total entre el dominio representado y el modelo. En ese caso, se trataría de una relación de isomorfía; mientras que -para emplear un término técnico de las matemáticas- en la representación científica se trata, en el mejor de los casos, de establecer un homomorfismo -una forma de relación más "débil" que la isomorfía, que produce una suerte de asimetría entre lo representado y el representante (este último es más rico en contenido "superfluo"). Más aún, en los casos más interesantes de representaciones científicas, el proyecto mismo de establecer una verdadera homomorfía entre representado y representante se vuelve irrealizable. Podemos buscar entre las dos partes relaciones funcionales que sean aún más débiles, si bien tienen que ser por lo menos informativas; este tipo de relación ha sido descrito a menudo como la 
"subsunción" (embedding) de un modelo empírico en un modelo matemático, pasando a ser el primero un "submodelo" del segundo."

No entraremos aquí en los detalles, muy técnicos, de las relaciones funcionales entre estructuras que pueden ser vistas como representaciones adecuadas en un contexto científico. Señalemos sólo que la idea esencial del representacionalismo consiste en concebir el conocimiento científico como la investigación de las relaciones funcionales (reconstruibles con toda la precisión necesaria) entre diferentes estructuras -relaciones que permitan hacer inferencias sobre la naturaleza del objeto estudiado (concebido ya de una cierta manera) partiendo de propiedades estructurales de los modelos utilizados para representarlo-.

Un tema esencial en el análisis de los fundamentos de la ciencia moderna -la cuestión de la naturaleza de la medición- constituye una línea de investigación particularmente fructífera en el seno del programa representacionalista. Medir objetos empíricos (tarea que, como se sabe, ha tenido un papel crucial en el desarrollo de la ciencia moderna y no sólo en las ciencias físicas) resulta ser un proceso de representación en el sentido preciso que acabamos de sugerir. ¿Qué es lo que hacemos cuando medimos los objetos de un dominio dado? La respuesta del representacionalismo es que se establece una relación de representación entre un dominio cualitativo dado y una estructura matemática (generalmente numérica). Por ejemplo, supongamos que queremos medir la estatura de los alumnos de una escuela; este conjunto de individuos es, primeramente, un objeto a representar como teniendo entre sus miembros relaciones "cualitativas" o "directamente observables" que se describen constatando que un alumno cualquiera es "más alto" o "tan alto" como otro. Ahora bien, cuando medimos la altura de los alumnos, lo que hacemos en última instancia no es más que asignar a este objeto "cualitativo" (el conjunto de alumnos articulado en torno a la relación observable de la altura) una estructura matemática que comprende números y la relación aritmética " $\geq$ ” entre estos números. Sin duda, éste que acabamos de dar es un ejemplo muy banal; sin embargo, la tesis del representacionalismo es que todas las formas de medición, incluso las más complejas conocidas en el dominio de las ciencias, consisten esencialmente en el mismo procedimiento. Lo que a veces se llama el proceso de "matematización" de una disciplina no es otra cosa que la investigación de las condiciones adecuadas que permiten establecer este tipo de relación funcional (llamada justamente "representación”) entre estructuras empíricas dadas y estructuras matemáticas adecuadas que las "representan" y nos permiten obtener información acerca de las primeras -informaciones que, sin las estructuras matemáticas correspondientes, no podrían ser obtenidas más que difícilmente o en absoluto, ya que el material cualitativo es generalmente mucho menos preciso y mucho más difícil de manipular conceptualmente-.

Para comprender mejor la importancia de este programa de reconstrucción del concepto de medición para la epistemología y la metodología general de la ciencia, ilustrémoslo mediante un ejemplo, aún bastante simple, pero más interesante que el precedente. Supongamos que a objetos físicos de tamaño medio queremos asignar números que expresen su peso (o más exactamente su masa) -que es justamente lo que se entiende por la expresión "medir el peso de los objetos"-. No tenemos un acceso observacional directo a estos números; todo lo que podemos constatar directamente es que hay objetos más pesados (en el sentido cualitativo) o tan pesados como los otros; además, también podemos constatar directamente que disponemos de un aparato (una balanza, por ejemplo) que nos permite reunir ("combinar" o "concatenar" son las expresiones técnicas habituales) dos objetos diferentes sobre un mismo platillo de balanza constituyendo así un tercer objeto que resulta, por así decirlo, de la combinación o concatenación de los otros dos. Se trata de constataciones puramente empíricas o "directamente observables". No se trata aún de números ni de "cantidades". Sin embargo, si la estructura constituida por el conjunto de objetos físicos, la relación entre ellos (que describimos como "ser tanto o más pesado que") y, finalmente, la operación de combinación consistente en

\footnotetext{
${ }^{4}$ Para una definición de esta noción de subsunción, ver, por ejemplo, el artículo de Suppes "Representation Theory and the Analysis of Science" [Teoría de la representación y el análisis de la ciencia], que contiene además una breve exposición de los objetivos generales del representacionalismo.
} 
ponerlos juntos sobre el mismo platillo de balanza -si esta estructura satisface algunas condiciones axiomáticas de naturaleza empírica-, se puede probar formalmente que existe una función numérica (una "magnitud"), que simbolizaremos por " $m$ " (la "masa"), que tiene la propiedad de asignar a cada objeto físico un número de tal modo que si un objeto $a$ es tanto o más pesado (en el sentido intuitivo, cualitativo) que otro $b$, entonces $m(a) \geq m(b)$, y si un objeto $c$ resulta de la combinación (empírica) de $a$ y $b$, entonces $m(c)=m(a)+m(b)$. Se puede también probar que esta función es unívoca, salvo por transformaciones de escala que es fácil formular. Se puede decir entonces que hemos representado la estructura empírica, constituida por objetos físicos más o menos pesados en relación los unos con los otros y combinables, mediante una estructura puramente matemática de números provista de las relaciones " $\geq$ ” y “+”. Brevemente, hemos representado los datos de la experiencia mediante una estructura matemática. He aquí un punto crucial para comprender en qué consiste la relación entre la experiencia y la matemática -lo que, como se sabe, es un tema central de la filosofía de la ciencia-. Ciertamente, la prueba de que es posible representar una determinada estructura empírica mediante una estructura matemática conveniente depende esencialmente de las condiciones axiomáticas que la estructura empírica debe satisfacer, y éstas no son, en general, condiciones fáciles de establecer.

La proposición que expresa que una estructura empírica dada satisface algunas condiciones que permiten probar la existencia y unicidad de una representación numérica apropiada para esa estructura es lo que se llama un teorema de representación. Una parte muy importante del programa representacionalista consiste justamente en establecer teoremas de representación para un gran número de conceptos científicos en todas las disciplinas (longitud, tiempo, masa, energía, entropía, carga eléctrica, etc. en física, y asimismo utilidad en economía u otros conceptos en psicología de la percepción o en teoría del aprendizaje). Estos resultados han sido expuestos por Suppes y sus colaboradores (D. Krantz, R.J. Luce y A. Tversky) en una obra monumental, Foundations of Measurement [Fundamentos de la medición]. El primer volumen (el más interesante desde el punto de vista epistemológico) apareció en 1971; los otros dos (muy técnicos) en 1987 y 1989.

En cierto sentido, se puede establecer una relación de filiación entre este programa representacionalista, que se apoya en la idea de que los verdaderos fundamentos del conocimiento científico están constituidos por entidades y operaciones directamente observables (siendo "derivados" los conceptos teórico-matemáticos más abstractos a partir de teoremas de representación) y el operacionalismo clásico de Bridgman. Sin embargo, mientras Bridgman y sus discípulos querían definir estrictamente los conceptos teóricos matematizados de la ciencia mediante operaciones de laboratorio, los representacionalistas actuales saben que tales "definiciones" no son en general posibles (o perjudicarían el desarrollo de las ciencias si se las postulara); consiguientemente, se limitan a estudiar las condiciones empíricas que permiten representar (lo que no equivale a "definir") los datos de la experiencia en una estructura teórico-matemática, que es en sí lógica y metodológicamente independiente de los datos empíricos. Se podría calificar el programa de teoremas de representación de "operacionalismo refinado".

A partir de los años setenta aparece otra corriente de la filosofía de la ciencia que también puede llamarse "representacionalista", aunque fue concebida de forma independiente. Se trata de los trabajos del físico Günther Ludwig (Alemania, 1918-2007) y de sus discípulos (todos ellos físicos teóricos). Desde un punto de vista histórico, el único trasfondo común de Ludwig y Suppes y sus sucesores es el uso del lenguaje de la teoría de conjuntos para construir las teorías científicas y para esclarecer sus relaciones con la experiencia preteórica. De hecho, Ludwig aplica los instrumentos de la teoría de conjuntos de una manera mucho más sistemática que Suppes y sus colaboradores, utilizando el aparato fundamental del programa Bourbaki. En el caso de Ludwig, el programa reconstructivo propuesto para las teorías físicas consiste en establecer los principios de correspondencia unívoca entre los conceptos teóricos y la base experimental específica de una teoría determinada, abandonando la idea de que pueda haber una base universal común a todo el conocimiento científico: cada teoría tiene su propia base experimental ya "preparada" (el Grundtext, en la terminología, bastante peculiar, de Ludwig), a partir de la cual ciertas correlaciones (Abbildungsprinzipien) son establecidas con ayuda del aparato 
matemático propio de la teoría en cuestión. Al igual que en otras formas de representacionalismo, estos principios de correlación son en general menos exigentes que los homomorfismos, pero deben ser formulados en el lenguaje de la teoría de conjuntos. Además, estos principios están siempre asociados a una estructura topológica que representa el aparato aproximativo que permite poner en relación el aparato matemático y el Grundtext. La idea de aproximación es, para Ludwig, una parte esencial e irreducible de toda verdadera teoría física: propugna una visión "aproximativista" del conocimiento científico, que será retomada en concepciones modelísticas posteriores. Ludwig aplicó sistemáticamente sus ideas a la reconstrucción de la mecánica cuántica y, de una manera menos sistemática, a otras teorías físicas más clásicas, como la mecánica newtoniana o la electrodinámica. Presentó su metateoría general en su principal obra, Grundstrukturen einer physikalischen Theorie (1978) [Las estructuras fundamentales de una teoría física]. Aun cuando sus trabajos comportan elementos originales y estimulantes para la reflexión epistemológica, tuvieron poca repercusión fuera del muy restringido círculo de físicos alemanes con intereses metodológicos. Tres factores pueden explicar esta carencia de divulgación: el estilo y la terminología de Ludwig son extremadamente particulares, a veces casi ininteligibles para el común de los filósofos; no intentó poner en relación su metateoría con otras concepciones contemporáneas de la filosofía de la ciencia, de las que parece ignorar casi todo; y (last but not least) ninguno de sus libros ha sido publicado en inglés...

\section{Las concepciones semanticistas}

En el mundo anglófono, se suele llamar semantic view ("concepción semántica") a un cierto número de enfoques que han marcado profundamente el desarrollo de la filosofía de la ciencia, sobre todo a partir de 1970, y que tienen sus orígenes (al menos en parte) en la obra de Suppes y de sus colaboradores inmediatos. El apelativo de "concepción semántica" puede ser, quizá, motivo de confusión, en la medida en que el rasgo común de esta familia de autores no es solamente señalar la importancia de los conceptos semánticos para analizar las teorías científicas (lo que ya habían señalado otros autores de la fase clásica de nuestra disciplina, como Carnap, Nagel, etc.), sino la predominancia absoluta de la visión semántica en detrimento de los análisis sintácticos, visión asociada a la preeminencia de la noción de modelo en sus diversas variantes. Calificaremos, pues, a estas concepciones de "semanticistas", más que de "semánticas".

Es bastante difícil circunscribir el conjunto de autores relevantes del "semanticismo", pues depende en gran medida de lo que se entienda por "predominancia de lo semántico" en la filosofía de la ciencia; así, según algunos comentadores, el estructuralismo metateórico, del que hablaremos más adelante, también pertenece a esta corriente, mientras que según otros no es así. Además, la dispersión geográfica de los grupos que han trabajado o aún trabajan en esta dirección es considerable. El "núcleo duro" del semanticismo se encuentra en Estados Unidos, pero en otros países existen grupos de investigadores que han contribuido de manera original a un programa que privilegia las consideraciones semánticas: es el caso de los estructuralistas en Alemania, Países Bajos y en los países iberoamericanos, de los polacos Marian Przełecki y Ryszard Wojcicki, de la escuela de Newton da Costa en Brasil, o del grupo reunido en torno a Maria Luisa Dalla Chiara y Giuliano Toraldo di Francia en Italia. Concentrémonos en las versiones más explícitas del semanticismo, tal y como ha sido expuesto principalmente en el compendio editado por Frederick Suppe en 1974, The Structure of Scientific Theories [La estructura de las teorías científicas]. Los tres autores más conocidos son Bas van Fraassen (Países Bajos/Canadá, nacido en 1941), el propio F. Suppe (Estados Unidos, nacido en 1940) y Ronald Giere (Estados Unidos, nacido en 1938), siendo el primero uno de los más influyentes filósofos de la ciencia de la última parte del siglo XX y principios del siglo XXI.

Bas van Fraassen reconoció en varios lugares su deuda intelectual con Patrick Suppes, quien para él tiene el mérito de haber liberado definitivamente a la filosofía de la ciencia de la obsesión de los análisis sintácticos y de haber abierto una perspectiva nueva y fecunda, definiendo las teorías científicas simplemente como clases de modelos. Sin embargo, van Fraassen diverge de Suppes por la manera 
concreta en que concibe un modelo científico: en lugar de definirlo como una estructura determinada mediante la teoría de conjuntos, van Fraassen propone concebir los modelos como "trayectorias" o "regiones" en un espacio de estados -idea que toma del lógico holandés E.W. Beth. A partir del comienzo de los años setenta, generaliza y desarrolla las ideas de Beth, ilustrándolas con ejemplos tomados de la física. Expone más sistemáticamente su metodología de reconstrucción de teorías y la epistemología asociada a ella algunos años más tarde en su libro The Scientific Image (1980) [La imagen científica].

La noción de espacio de estados proviene directamente de la física. Recordemos que en termodinámica, por ejemplo, se considera que el estado de un sistema físico está completamente determinado por una tríada ordenada de números reales $\langle p, v, t\rangle$, que representan respectivamente los valores de las magnitudes presión, volumen y temperatura. En general, en las teorías físicas, los estados de un sistema físico son identificados por puntos en un sistema de coordenadas con tantas dimensiones como componentes determinan el estado. A cada tipo de sistema físico corresponde así un espacio de estados, que es el conjunto de todas las secuencias posibles de $n$ elementos $(n=3$ para el ejemplo de la termodinámica). Los estados posibles del sistema físico son "puntos" en este "espacio". La tarea de los axiomas propios de una teoría dada consiste entonces en imponer constricciones al conjunto de secuencias lógicamente posibles, fijando las secuencias que son aceptables y las que no lo son. Las secuencias aceptables representan los modelos admitidos por la teoría. La analogía con el procedimiento de Suppes es evidente: una teoría física no es de hecho nada más que un conjunto constituido por un gran número de modelos; la diferencia reside en el hecho de que los modelos son concebidos en este caso como trayectorias (o regiones) en un espacio de estados.

Lo que distingue la reconstrucción de las teorías en términos de un predicado conjuntista o de la determinación de un espacio de estados es más metodológica que epistemológica. Se trata de dos procedimientos para hacer más transparente la estructura interna de las teorías científicas, sin que haya una divergencia profunda en cuanto a la "esencia" de las teorías, que es siempre la misma, a saber, un conjunto de modelos. Cada uno de estos procedimientos tiene sus ventajas y sus inconvenientes. El método de espacios de estado es ciertamente más cercano a la manera en que las teorías se presentan en los manuales de física moderna, al menos en el caso de la mecánica clásica y la mecánica cuántica. Esto resulta menos evidente en el caso de otras teorías, incluso en física (por ejemplo, en el caso de la teoría de la relatividad, como van Fraassen lo reconoció más tarde) y sobre todo no lo es en absoluto en el caso de las teorías cuyos conceptos fundamentales no son magnitudes, sino relaciones o propiedades "cualitativas" -como en muchas teorías de la química, la biología y las ciencias sociales-. Por esta razón, el método de definición de los modelos por medio de un predicado conjuntista parece ser más universalmente aplicable. Tiene además la ventaja de hacer inmediatamente transparente la estructura formal del "compromiso ontológico" de cada teoría, caracterizando explícitamente sus dominios de entidades básicas en las condiciones que define el predicado conjuntista (como ya hemos visto en el caso de "la teoría de la familia"). Pero se trata más de una diferencia en la práctica reconstructiva de los filósofos de la ciencia que de una diferencia de principio.

Van Fraassen se muestra más tajante que Suppes y sus colaboradores en el desarrollo de la interpretación epistemológica acerca de la manera en que los modelos que constituyen una teoría científica se relacionan con la realidad empírica. Hemos visto que en Suppes y los representacionalistas más próximos a él, se detecta implícitamente una suerte de operacionalismo sofisticado. En cambio, la posición epistemológica de van Fraassen es más explícita y radical. Defiende una variante particular de instrumentalismo que describe como un "empirismo constructivo" y que es completamente antirrealista. El empirismo constructivo tiene consecuencias notables sobre un gran número de temas tradicionales importantes de la filosofía de la ciencia, tales como la causalidad, la naturaleza de la explicación científica y la de las leyes o de la base empírica. Van Fraassen explicitó estas consecuencias no sólo en The Scientific Image, sino en obras posteriores como Laws and Symmetry (1989) [Leyes y simetría].

Distingue en cada teoría la parte "teóricamente pura" de aquella que concierne a las aserciones empíricas. La parte pura determina el tipo de modelos con los cuales el científico se compromete a 
trabajar; la parte empírica añade aquellos dominios de la experiencia a los que presuntamente son aplicables los modelos de la teoría. Esto recuerda mucho la distinción introducida por Adams (ver supra, p. 79) entre la clase $M$ de modelos y la clase I de aplicaciones intencionales. Pero van Fraassen evita la dificultad de la "justificación circular" que hemos constatado al exponer las ideas de Adams, señalando que estas aplicaciones son concebidas como subestructuras de las estructuras que constituyen los modelos. Estas subestructuras representan nuestras observaciones empíricas, en tanto que el contenido empírico de la teoría consiste en la aserción de que dichas subestructuras pueden ser efectivamente subsumidas en un modelo completo de la teoría, determinado por las leyes. En este proceso de subsunción, la teoría postula la existencia de entidades no observables, puramente teóricas, las cuales, en su interacción (postulada por las leyes de los modelos teóricos) con las entidades observables a las que se refieren las subestructuras observables, explican los fenómenos que de hecho observamos. Si la aserción según la cual las subestructuras observables pueden ser subsumidas en un modelo de la teoría queda confirmada por nuestras observaciones y experimentos, en tal caso podemos decir que hemos "salvado los fenómenos" y que la teoría es empiricamente adecuada. La tarea de las ciencias teóricas consiste en mostrarlo.

Para van Fraassen, es necesario distinguir entre la verdad de una teoría y su adecuación empírica. No es un "instrumentalista puro", puesto que admite un sentido (lógicamente impecable) en que se puede suponer que los modelos de una teoría son literalmente "verdaderos en relación con el mundo real". Pero es un instrumentalista en un sentido más amplio, ya que niega que se pueda justificar científicamente esta suposición. Además, la considera como desprovista de pertinencia para la aceptación de una teoría científica, en tanto los modelos están siempre subdeterminados en relación con la observación (de hecho, retoma la tesis de Quine de la subdeterminación empírica de las teorías, aunque dentro de un marco conceptual distinto). La única cosa que cuenta para la buena marcha de la ciencia es mostrar que sus teorías son empíricamente adecuadas. Es por esta razón que van Fraassen define su posición como una suerte de empirismo constructivo: "Empleo el adjetivo constructivo, para indicar mi concepción según la cual la actividad científica es una actividad de construcción y no de descubrimiento: una construcción de modelos que deben ser adecuados a los fenómenos, y no un descubrimiento de la verdad de lo que es inobservable" (van Fraassen 1980, p. 5).

Evidentemente, la epistemología del empirismo constructivo depende esencialmente de la noción de observabilidad, ya que son las subestructuras observables de los modelos lo que nos permite decidir si una teoría propuesta es empíricamente adecuada o no. Nos hace falta un criterio preciso para distinguir los objetos observacionales de los objetos no observacionales; si no, las tesis del empirismo constructivo serían vagas y, sobre todo, no se sabría cómo aplicarlas a la reconstrucción efectiva de teorías concretas. Ahora bien, son ampliamente conocidas las grandes dificultades que tuvieron los filósofos de la ciencia de la fase clásica para formular una distinción tajante entre el nivel observacional y el nivel teórico en el discurso científico, así como las críticas decisivas de los filósofos historicistas en contra de la idea de conceptos puramente observacionales. Van Fraassen es más o menos consciente de estas dificultades (aun cuando, sorprendentemente, no las discute muy a fondo) y propone un criterio de observabilidad antropomórfico. Este criterio pretende tener un valor estrictamente epistemológico (sobre lo que nosotros, en tanto que miembros de la especie Homo sapiens, estamos o no capacitados para saber) y no ontológico: en tanto que organismos biológicos dotados de "instrumentos de detección" particulares (nuestros órganos sensoriales), hay cosas que podemos observar y otras que definitivamente son inobservables -y de estas últimas hay que abstenerse de afirmar nada-.

El criterio de observabilidad propuesto por van Fraassen no es muy preciso (un objeto que, por su naturaleza, es detectable solamente mediante un microscopio electrónico idebe ser considerado "observable para el Homo sapiens"?); pero el problema principal es que, en las teorías un tanto desarrolladas, es poco verosímil postular que las subestructuras destinadas a constituir la base empírica contengan nociones puramente observacionales en el sentido de van Fraassen. Se trata más bien, como él mismo reconoció en Laws and Symmetry, de "modelos de datos" (expresión que toma de Suppes), es decir, de estructuras conceptuales ya muy sofisticadas en relación con lo que se puede imaginar como 
"directamente observable". Por ejemplo, las subestructuras que constituyen la base empírica de la mecánica clásica son, sin duda, subestructuras cinemáticas, lo que quiere decir que están constituidas, entre otras cosas, por la noción de tiempo continuo y de distancia, que es una magnitud dos veces diferenciable en el sentido del cálculo diferencial; sería muy extraño suponer que las nociones de continuidad y de diferenciabilidad aquí requeridas correspondan al sentido antropomórfico de observabilidad postulado por van Fraassen. En breve, con su empirismo constructivo, este investigador presentó la versión más fuerte y la más sistemáticamente desarrollada de antirrealismo de los últimos decenios de la filosofía de la ciencia; sin embargo, en la medida en que la noción de observabilidad que le es crucial continúe tan poco articulada, la base epistemológica del empirismo constructivo seguirá siendo ella misma problemática.

Frederick Suppe es otro de los representantes de la versión semanticista del modelismo, que intenta encontrar una "vía intermedia" entre el realismo y el antirrealismo en epistemología. Ya en 1967, en su tesis doctoral intitulada The Meaning and Use of Models in Mathematics and the Exact Sciences [El significado y uso de los modelos en matemáticas y las ciencias exactas], tomó de Suppes la noción de modelo, aunque reformulándola en términos de espacios de estados (independientemente de van Fraassen). De manera más sistemática, Suppe desarrolló sus ideas en su obra principal, The Semantic Conception of Theories and Scientific Realism (1989) [La concepción semántica de las teorías y el realismo científico]. El aparato formal de reconstrucción de las teorías propuesto por Suppe es casi idéntico al de van Fraassen: una teoría no es otra cosa que un sistema relacional consistente en dos componentes, un dominio que contiene todos los espacios de estados lógicamente posibles de los sistemas estudiados, y un cierto número de relaciones entre estados, determinadas por los axiomas de la teoría, que especifican las trayectorias y regiones físicamente posibles. Para Suppe, "físicamente posible" quiere decir "causalmente posible".

Usando una terminología análoga a la de Adams, Suppe describe la base empírica de una teoría como un "alcance pretendido" (intended scope) de la experiencia, constituido por lo que él llama los "datos duros" (hard data). Si bien concibe la parte puramente teórica de una teoría de una manera muy similar a la de van Fraassen, difiere, en cambio, en la interpretación de los "datos duros" que constituyen el alcance pretendido. No es necesario interpretarlos en el sentido de "directamente observable". Según Suppe, la dicotomía "observacional/teórico" no tiene papel alguno en la epistemología. Debe ser reemplazada por una distinción, relativa a una teoría dada, entre, por un lado, los datos no problemáticos para la teoría en cuestión (los datos "duros") y, por otro, las hipótesis teóricas características de la teoría, que son confrontadas con los datos no problemáticos.

Los "datos duros" de Suppe son relativos no sólo a una teoría particular, sino también en el sentido de que, incluso dentro de la teoría en cuestión, pueden ser revisados en todo momento. La razón es que las estructuras que constituyen la base empírica de una teoría son siempre estructuras conceptuales muy abstractas e idealizadas. Suppe asigna un lugar importante a la noción de idealización en las ciencias empíricas. Los datos son obtenidos mediante un proceso muy complejo, seleccionando algunos parámetros de entre otros para la descripción de los fenómenos (lo que constituye la esencia del proceso de la idealización); esta selección siempre puede ser puesta en duda en el seno mismo de la teoría si las cosas no van como se esperaba o si hemos sido capaces de elaborar métodos de análisis más avanzados.

En la concepción de Suppe, una teoría es empiricamente verdadera (o adecuada) si los datos duros coinciden con los modelos de la teoría o, más exactamente, si los sistemas físicos que constituyen el alcance pretendido coinciden con los espacios de estados causalmente posibles determinados por las leyes teóricas (admitiendo siempre algunas idealizaciones). Pero dado que hemos visto que estos "datos duros" son, ellos mismos, estructuras abstractas e idealizadas, construidas, no podemos afirmar que una teoría empíricamente verdadera en este sentido refleje la "realidad tal cual es". La epistemología de Suppe es en este punto muy similar a la de van Fraassen. Sin embargo, difiere en un aspecto importante. La condición de adecuación entre datos y espacios de estados causalmente posibles es, de hecho, sólo una condición necesaria, pero no suficiente por sí misma, para el buen funcionamiento de una teoría empírica. Suppe añade lo que califica de requisito "antinominalista": los parámetros 
seleccionados por la descripción de los datos duros deben corresponder a clases naturales, y no ser escogidos ad hoc. Y la noción de "clase natural" no es antropomórfica; muy al contrario, se supone que corresponde a aspectos de la realidad independientes de la observación y de la teorización humanas. El postulado según el cual los parámetros seleccionados para determinar los datos corresponden a clases naturales nos permite justificar la suposición (contrafáctica) según la cual "el mundo real sería exactamente como lo describe la teoría, caso de que las únicas clases naturales pertinentes fueran las que corresponden a los parámetros escogidos", es decir, si pudiéramos eliminar con éxito toda idealización (lo que siempre resulta imposible en la práctica). Es por esta razón que Suppe describe su propia posición como un "cuasi realismo" (en contraposición con el empirismo radical de van Fraassen). Sin embargo, esta posición tiene también su propia dificultad, análoga a la que encontramos en van Fraassen en relación con la observabilidad: ¿de qué criterios disponemos para distinguir los parámetros que designan clases naturales de aquellos que no las designan? Parece que los únicos criterios factibles en una disciplina científica cualquiera (si no queremos apoyarnos en una metafísica esencialista de clases naturales) son los que la teoría misma proporciona. Por tanto, parece que el "cuasi realismo" de Suppe es aún más débil de lo que el prefijo "cuasi" connota y que corre el riesgo de ser simplemente un pseudorrealismo.

El tercer protagonista de las concepciones semanticistas, Ronald Giere, ha desarrollado su propia versión de esta concepción en el marco de un programa metacientífico más amplio que el de los dos autores precedentes -el de una perspectiva cognitivista general-. En el curso de los años, resultó evidente para Giere que no existe ninguna diferencia esencial entre la filosofía de la ciencia y las ciencias cognitivas, teniendo ambas disciplinas el mismo objeto de estudio y (potencialmente) los mismos métodos de investigación. (Se podría interpretar esta fusión como una manera concreta de comprender el proyecto de Quine de una "epistemología naturalizada".) Esta tendencia hacia el cognitivismo ya es remarcable en su primera obra sistemática, Explaining Science (1988) [Explicando la ciencia], pero se hace aún más evidente en su segundo tratado, Cognitive Models of Science (1992) [Modelos cognitivos de la ciencia].

$\mathrm{Al}$ igual que en los otros autores a quienes nos hemos referido en esta presentación, el punto de partida de Giere descansa en el "núcleo duro" de una teoría como conjunto de modelos. Sin embargo, a diferencia de los otros representantes del modelismo, él no quiere limitarse a una reconstrucción formal determinada de los modelos en cuestión -ni en tanto que estructuras conjuntistas, ni en tanto que espacios de estados. Su concepto de "modelo teórico" es extremadamente amplio (y también hay que decirlo: peligrosamente vago). Los modelos que constituyen las teorías científicas pueden ser, según él, todo tipo de entidades abstractas que normalmente son descritas por medio de un lenguaje más o menos técnico, pero que a veces también pueden ser determinadas por medios no lingüísticos (por ejemplo, gráficas, esquemas, mapas, etc.). La sola característica común a todos los modelos tomados en este sentido es que se trata de entidades abstractas, no empíricas o "mentales", que son construidas y validadas socialmente por una comunidad de científicos.

Ahora bien, la función de estas entidades abstractas que llamamos "modelos teóricos", al menos en las ciencias empíricas, consiste (como en otras concepciones modelísticas) en una representación de ciertos aspectos de sistemas reales mediante algunas hipótesis teóricas que vinculan los modelos con la realidad y que afirman que un sistema real dado es parecido, en ciertos aspectos y en cierta medida, a un modelo construido en el discurso científico. Así, por ejemplo, por un lado tenemos un sistema real como el constituido por la Tierra, el Sol y la Luna, y, por otro, un modelo de la mecánica newtoniana tal como el descrito por tres partículas que se atraen mutuamente con una fuerza proporcional a la inversa del cuadrado de la distancia. La hipótesis que lanza aquí el científico es que ciertos aspectos del sistema Sol-Tierra-Luna (por ejemplo, sus posiciones y sus aceleraciones respectivas) son muy próximos al modelo de tres partículas con el tipo de fuerza descrito.

Al igual que en Suppe, el instrumento conceptual de la idealización o la aproximación tiene un papel esencial en la concepción de Giere acerca de la relación entre los modelos teóricos y la realidad que se supone representan. Ciertamente, si no estamos satisfechos con el grado de aproximación y/o de idealización constatado en la relación entre el sistema estudiado y el modelo propuesto, podemos 
cambiar de modelo, y construir otro tomando en cuenta algunos aspectos del sistema real antes ignorados y que nos conduce a grados de aproximación más adecuados. Pero es utópico suponer que podremos alcanzar algún día una correspondencia exacta entre el modelo y la realidad. Esto es así en virtud de la naturaleza misma tanto del modelo como del objeto representado.

De este pluralismo y este "imperfeccionismo" de la relación entre los modelos y los sistemas reales, Giere extrae una consecuencia más general sobre la naturaleza de las teorías científicas -consecuencia que no es el resultado de una argumentación lógica estricta, sino que es, a lo sumo, sugerida por la situación descrita: las teorías científicas son entidades irremediablemente vagas, sus condiciones de identidad no pueden ser fijadas con precisión-. Por ejemplo, es imposible decir lo que tienen realmente en común todos los modelos newtonianos. Tienen, ciertamente, un "aire de familia", pero esta semejanza es insuficiente para proporcionarnos una definición formal y unívoca de la noción general de "modelo newtoniano". La cuestión de saber si dos modelos propuestos para estudiar un mismo sistema pertenecen o no a la misma familia (por ejemplo, la "familia newtoniana") es decidida exclusivamente por los miembros de la comunidad científica en un momento dado. Esto no significa que una semejanza objetiva pueda ser juzgada de una manera correcta o incorrecta, sino que el conjunto de juicios de los científicos determina la cuestión de saber si la semejanza es suficiente. Esto es un aspecto del hecho de que las teorías no sólo son construidas, sino que además son "socialmente construidas" (Giere 1988, p. 86). Claramente, Giere introduce así un fuerte elemento pragmático en su concepción de la identidad de las teorías científicas.

Sobre la base de esta concepción pragmática (que se aleja de los postulados más tradicionales del semanticismo), Giere adopta un tipo particular de "realismo", al cual da el nombre de "realismo constructivo" -por oposición al "empirismo constructivo" de van Fraassen-. Se trata de una concepción donde la dimensión "constructivista" es mucho más fuerte que la dimensión "realista”. En efecto, para Giere, la ciencia tiene un aspecto esencialmente constructivo -la construcción de modelos-, y siempre habrá modelos diferentes para representar de manera alternativa el mismo sistema real. Existen, sin duda, modelos mejores que otros, pero no podemos especificar esta diferencia apelando sólo a la "realidad". No hay nada en el mundo que determine los aspectos "verdaderamente esenciales" que uno debe representar por medio de los modelos, ni el grado de adecuación de una representación propuesta. La especificación de la diferencia entre "buenos" modelos y modelos "menos buenos" es una cuestión que remite en última instancia a intereses humanos y estos intereses no son sólo epistémicos, sino también prácticos. Esta constatación nos lleva a un cierto relativismo, pero no a un relativismo radical: para poner un ejemplo del propio Giere, podemos circular por Nueva York de una manera más o menos adecuada con mapas diferentes de la ciudad, pero no con un mapa de San Francisco. Esta diferencia nos permite comprender, según Giere, que la dosis de relativismo inherente a la pluralidad y a la imperfección substancial de los modelos, en tanto que representaciones de la realidad, es compatible con una cierta dosis de realismo: hay algo en la realidad que hace que dos planos diferentes de Nueva York puedan ser utilizados para circular por la ciudad, y que al mismo tiempo hace que un plano de San Francisco sea completamente inservible.

Ahora bien, el problema con este tipo de realismo "moderadamente relativista" es que, en un análisis más fino, parece muy próximo al antirrealismo (à la van Fraassen, por ejemplo). Los instrumentalistas o los antirrealistas pueden muy bien aceptar que haya modelos más o menos adecuados a un cierto dominio de la experiencia, mientras que otros no lo son. Pero si no se es un poco más preciso que Giere sobre la naturaleza de los sistemas reales que determinan, por medio de la relación de "semejanza suficiente", que ciertos modelos son válidos y otros no, y si el propio criterio de "semejanza suficiente" se deja al arbitrio de la comunidad científica del momento, entonces el instrumentalista no se dejará impresionar. Como en el caso de Suppe, aunque por otras razones, el "realismo constructivo" de Giere se revela al fin peligrosamente próximo a un pseudorrealismo o a un verdadero instrumentalismo. 


\section{El estructuralismo metateórico}

Sin duda, la concepción estructuralista de las teorías científicas forma parte de los enfoques que hemos englobado bajo la etiqueta común de "modelismo", ya que concede un lugar preeminente a la noción de modelo y está fuertemente inspirada en los trabajos de la Escuela de Stanford. No puede ser considerada como una simple continuación del conjuntismo suppesiano, ya que ha desarrollado un aparato conceptual de análisis de las ciencias empíricas mucho más complejo y sofisticado que el de Suppes y sus discípulos, intentando incorporar de forma sistemática aspectos importantes del análisis propuesto por autores historicistas como Kuhn y Lakatos (especialmente el primero, que reconoció -en un artículo de 1976 para la revista Erkenntnis o, más tarde, en una entrevista concedida un poco antes de su muerte y publicada póstumamente- sus afinidades profundas con el estructuralismo, a pesar de las diferencias aparentemente insalvables "en la superficie").

Antes de que comencemos a exponer las ideas principales del estructuralismo que hemos calificado de "metateórico", se hacen necesarias algunas precisiones históricas y terminológicas para evitar enojosos malentendidos. El estructuralismo del que aquí se trata no tiene mucho que ver con el estructuralismo francés -corriente que marcó profundamente la filosofía y las ciencias humanas en Francia durante los años sesenta y setenta-. También tiene poco que ver con la manera "estructuralista" de practicar la lingüística y la interpretación literaria, extendida hoy un poco por todos los lugares del mundo. El único "estructuralismo" con el cual la concepción estructuralista de la filosofía contemporánea de la ciencia tiene una afinidad metodológica es con el practicado en el estudio de los fundamentos de la matemática, sobre todo (aunque no únicamente) en la versión puesta en práctica por el grupo llamado Bourbaki sobre la reconstrucción conjuntista de las teorías matemáticas. Habrán de pasar varios años antes de que el programa de investigación metateórica que examinamos aquí lleve su nombre actual. El pionero de estos estudios, Joseph D. Sneed (Estados Unidos, nacido en 1938), no atribuye ninguna denominación específica a la concepción formulada en su obra fundacional, The Logical Structure of Mathematical Physics (1971) [La estructura lógica de la física matemática]; solamente caracteriza una parte de sus resultados como una "concepción de Ramsey modificada" (emended Ramsey view). Sin embargo, esta descripción resulta completamente inadecuada o, en el mejor de los casos, se refiere a un aspecto bastante secundario de su concepción. El otro "fundador" del estructuralismo en filosofía de la ciencia, Wolfgang Stegmüller (Austria, 1923-1991), en su primera obra, Theorienstrukturen und Theoriendynamik (1973) [Estructura y dinámica de teorías], consagrada a la nueva metateoría, retoma y desarrolla la concepción de Sneed, caracterizándola como una "concepción no enunciativa" (non-statement view) de las teorías científicas -denominación un poco menos inadecuada que la precedente, pero aún bastante incompleta-. Es sólo hacia fines de los setenta que el lógico y filósofo israelí Yehoshua Bar-Hillel sugirió a Stegmüller describir su concepción como un tipo de estructuralismo, en razón de su semejanza (metodológica) con el estructuralismo del grupo Bourbaki en matemáticas. Stegmüller aceptó esta sugerencia y tituló su nueva obra programática The Structuralist View of Theories (1979) [La concepción estructuralista de las teorías]. Si bien esta denominación no es totalmente satisfactoria, pues se presta a confusión y no captura todos los aspectos relevantes de esta nueva corriente, es sin embargo la que se ha acabado imponiendo y la que vamos a mantener aquí.

La aparición del libro de Sneed pasó casi totalmente desapercibida por su complejidad y la novedad de sus ideas, así como por el nivel altamente técnico del aparato conceptual utilizado, pero también por su estilo expositivo, que se parece más a un informe de investigación que a un ensayo destinado a una amplia audiencia. Sin embargo, Stegmüller, entonces conocido como representante de la filosofía de la ciencia clásica y en un momento de cuestionamiento de la misma a causa de las dificultades inherentes a la "teoría de los dos niveles" (y a causa también de la lectura de Kuhn), comprende el potencial de la obra de Sneed para resolver ciertas dificultades de principio que le preocupan. En Theorienstrukturen und Theoriendynamik, expone las ideas de Sneed de una manera más accesible y 
propone aplicarlas al desarrollo de una nueva concepción de las teorías científicas: ésta debe permitir que se salga del callejón sin salida al que la concepción clásica había conducido a la filosofía de la ciencia, y "reconstruir racionalmente" las nociones y las tesis de Kuhn (y, en una cierta medida, también las de Lakatos). La comunidad de epistemólogos, "clásicos" o "historicistas", se empieza a interesar entonces por la nueva metodología propuesta por Stegmüller.

De 1974 a 1976, Sneed colaboró estrechamente con Stegmüller en el instituto que éste dirigía en Munich, y con otros dos investigadores, Wolfgang Balzer (Alemania, nacido en 1947) y el autor de estas líneas (Venezuela/España, nacido en 1946). Así toma forma el "programa estructuralista de reconstrucción de las ciencias empíricas”, que se desarrolla y evoluciona gradualmente a partir de mediados de los años setenta para llegar a su punto culminante en la obra de Balzer, Moulines y Sneed, An Architectonic for Science (1987) [Una arquitectónica para la ciencia]. Tomaré esta obra como referencia para exponer aquí los principales elementos del estructuralismo. El programa estructuralista puede ser caracterizado como un "programa abierto" en el sentido de que muchos de sus conceptos, principios y métodos han sido ampliados, modificados, revisados después de su aparición, conforme se iban encontrando dificultades internas (más o menos técnicas) en el aparato conceptual, malentendidos en su interpretación epistemológica general o insuficiencias en su aplicación a la reconstrucción de teorías concretas de disciplinas empíricas. Algunos de los desarrollos posteriores más importantes están reunidos en la compilación de Balzer y Moulines, Structuralist Theory of Science. Focal Issues, New Results (1996) [La teoría estructuralista de la ciencia. Temas centrales, nuevos resultados]. Muchos autores de todo el mundo han contribuido luego al programa estructuralista. Hay que decir que los "estructuralistas" han tomado siempre muy en serio la conminación a aplicar su metateoría a cuantos casos concretos y reales de teorías científicas sea posible. (En el momento en que escribo estas líneas, al menos unas cincuenta teorías de disciplinas científicas - de la física a la sociología pasando por la química, la biología, la psicología y la economía- han sido reconstruidas con todos los detalles y la precisión necesaria. $)^{5}$

El aparato conceptual utilizado por los estructuralistas en sus análisis y sus reconstrucciones es más complejo que el de las otras concepciones modelísticas aquí discutidas. Este aparato emplea instrumentos formales bastante técnicos de la teoría de conjuntos y de otras ramas de las matemáticas. Lejos de provenir (como lo han sugerido algunos críticos) del deseo absurdo de hacer más complicadas las cosas de lo que lo son, esta complejidad formal proviene del reconocimiento de que los objetos de estudio (las teorías científicas y las relaciones que tienen entre ellas) son en general entidades ellas mismas muy complejas y que, si uno no diera cuenta de esta complejidad, se caería en una visión demasiado simplista o vaga de la estructura de las ciencias empíricas. Vamos a intentar sin embargo echar un vistazo a los elementos esenciales de la metodología estructuralista sin sobrecargarnos de detalles formales y quedándonos al nivel más "intuitivo" posible.

El estructuralismo debe su nombre a la idea básica, común a los otros enfoques modelísticos, según la cual la manera más conveniente de interpretar la "esencia" de una teoría científica no consiste en recurrir a un conjunto de proposiciones, sino a la agrupación de tipos diferentes de estructuras complejas, ellas mismas compuestas de estructuras más simples. Las unidades estructurales más simples que constituyen una teoría son los modelos, concebidos (en la tradición Tarski-McKinsey-Suppes) como tuplas de la forma:

$$
\left\langle D_{1}, \ldots, D_{m}, R_{1}, \ldots, R_{n}\right\rangle,
$$

donde las $D_{i}$ representan los "dominios base" y las $R_{\mathrm{j}}$ son relaciones construidas (en el sentido de la teoría de conjuntos) sobre los dominios base. Estos fijan "la ontología", es decir, los conjuntos de objetos admitidos por la teoría como siendo "reales". Las relaciones fijan las relaciones admitidas entre

\footnotetext{
${ }_{5}^{5}$ Para una lista detallada (aunque no exhaustiva) de contribuciones al programa estructuralista, ver la bibliografía establecida por W. Diederich, A. Ibarra y Th. Mormann, "Bibliography of Structuralism" [Bibliografía del estructuralismo], actualizada y ampliada por C. Abreu, P. Lorenzano y C. U. Moulines en esta misma revista (Abreu, Lorenzano \& Moulines 2013). Un compendio de reconstrucciones de teorías particularmente características de disciplinas diversas ha sido reunido por W. Balzer, C. U. Moulines y J. D. Sneed en Structuralist Knowledge Representation: Paradigmatic Examples [Representación estructuralista del conocimiento: ejemplos paradigmáticos].
} 
los objetos de estos diversos conjuntos; en las teorías un tanto "avanzadas" estas relaciones serán generalmente funciones numéricas, es decir, magnitudes. Los dominios y las relaciones específicas de una teoría particular son caracterizadas por un cierto número de condiciones formales que determinan el "marco conceptual" de la teoría; por ejemplo, podremos especificar que el dominio $D_{1}$ debe ser un conjunto finito de objetos, mientras que el dominio $D_{2}$ debe ser un continuo, que la relación $R_{1}$ debe ser una relación simétrica y transitiva o que la relación $R_{2}$ debe ser una función dos veces diferenciable sobre los números reales, y así sucesivamente. Cuando todas estas condiciones formales del "marco conceptual" son satisfechas, se dice que la estructura en cuestión es un modelo potencial de la teoría. Es "potencial" en el sentido de que fija un marco posible para concebir la realidad sin que tengamos aún la garantía de que sirva para representar algunos aspectos substanciales de ésta, construir explicaciones o hacer predicciones. Las condiciones estipuladas son puramente a priori. Para que la estructura en cuestión sea no sólo un modelo potencial, sino también un modelo actual, es necesario que, además de sus "condiciones-marco", satisfaga "leyes de la naturaleza", es decir, ciertos axiomas en el sentido propio del término. Puesto que el estructuralismo no defiende una concepción proposicional de las teorías, no considera esencial decidir qué formulación concreta de estos axiomas se debe escoger; habrá siempre un número indeterminado de conjuntos diferentes de axiomas propios que determinan la misma clase de modelos actuales; sin embargo, es importante, de una manera o de otra, fijar la clase de modelos con los cuales se quiere "decir alguna cosa substancial sobre el mundo".

La identificación de una teoría cualquiera consiste pues, primeramente, en fijar el conjunto de sus modelos potenciales y actuales. Hasta ahí la metodología reconstructiva del estructuralismo no se distingue esencialmente de los otros enfoques modelísticos, sobre todo de la metodología de la escuela de Stanford (si bien la primera subraya la necesidad de distinguir claramente entre lo que corresponde al marco conceptual a priori y lo que corresponde a las leyes sustanciales con contenido empírico). Sin embargo, esto no es más que el primer paso en la identificación de una teoría. Una tesis central del estructuralismo es justamente que las teorías empíricas (al contrario de las teorías puramente matemáticas) están generalmente constituidas por más elementos que los modelos potenciales y actuales. Hay al menos otros cuatro componentes, que son esenciales a la correcta comprensión de su funcionamiento, para determinar la identidad de una teoría.

1) Los modelos (potenciales o actuales) de una teoría cualquiera no aparecen aislados los unos de los otros: están vinculados por ciertas condiciones (generalmente implícitas) que constriñen los componentes de cada modelo (por ejemplo los valores de una función determinada) en función de componentes de otros modelos. Desde un punto de vista formal, se trata aquí de condiciones de segundo orden (de condiciones sobre los modelos y no de condiciones en los modelos). El término utilizado para estas condiciones es el de "condiciones de ligadura" (constraints en inglés, Nebenbedingungen en alemán). Ejemplos de tales condiciones de ligadura, familiares a estudiantes de física, son los principios de invariancia. Las combinaciones de modelos que no satisfacen estas restricciones o condiciones de ligadura quedan simplemente excluidas de la identidad de la teoría.

2) Las teorías mismas no son entidades aisladas las unas de las otras. Esto quiere decir que los modelos de una teoría están vinculados no sólo a otros modelos de la misma teoría, sino igualmente a modelos de teorías diferentes. Por ejemplo, es esencial saber, para el buen funcionamiento de la termodinámica, que sus modelos empíricamente válidos están vinculados de una manera determinada a modelos de la hidrodinámica. Estos vinculos interteóricos (links en inglés, Bände en alemán) pertenecen asimismo a la "esencia" de una teoría empírica.

3) En general, hay que distinguir dos niveles conceptual y metodológicamente diferentes en el seno de una misma teoría: el de los conceptos que son específicos de la teoría en cuestión y que pueden ser determinados solamente si se presupone la validez de la teoría, y el de aquellos conceptos que provienen "de fuera", generalmente de otras teorías "subyacentes”. Los primeros pueden ser calificados de T-teóricos en relación con la teoría 
$\mathrm{T}$, los segundos de T-no-teóricos. La tupla de conceptos T-no-teóricos constituye evidentemente una subestructura de un modelo potencial de T. El conjunto de estas subestructuras recibe el nombre de "conjunto de modelos potenciales parciales". Desde un punto de vista intuitivo, este conjunto constituye el marco conceptual de los datos que supuestamente confirman o desconfirman la teoría, dado que los conceptos que los constituyen son independientes de la teoría en cuestión. Esta distinción entre dos niveles conceptuales podría ser interpretada como una reminiscencia de la teoría de los dos niveles (teórico/observacional) de la filosofía clásica de la ciencia. Pero tiene, de hecho, un sentido completamente diferente: la distinción estructuralista entre conceptos $T$ teóricos y T-no-teóricos no es meramente semántica (y menos aún sintáctica); no apela a la posibilidad de una "observación directa" y tampoco es universal (es decir, la misma para todas las teorías científicas, como cuando se postula un "lenguaje observacional" común a todas las ciencias) sino "local", es decir, relativa a cada teoría. Lo que es Tteórico con respecto a una teoría puede ser T-no-teórico con respecto a otra. Por ejemplo, las magnitudes dinámicas, masa y fuerza, son T-teóricas en la mecánica, pero Tno teóricas en la termodinámica.

4) Toda teoría empírica, que quepa tomar en serio, es aproximativa. La aproximación puede ser cualitativa o cuantitativa y puede variar según el tipo de aplicación que se tenga en mente; pero en ningún caso es un "modelo exacto" lo que se utiliza para representar la experiencia, sino más bien un conjunto "borroso" de modelos, determinado dentro de unos límites admisibles de "emborronamiento". Para definir estos "conjuntos borrosos", los estructuralistas (inspirados en este punto por Ludwig) recurrieron a la noción topológica de uniformidad. Estas uniformidades de modelos también pertenecen, de modo esencial, a la identidad de las teorías empíricas.

La combinación coherente de los seis conjuntos de estructuras que acabamos de describir (el conjunto de los modelos potenciales, el de los modelos actuales, el de los modelos potenciales parciales, el de las condiciones de ligadura, el de los vínculos interteóricos y la estructura de aproximación determinada por una uniformidad) constituye lo que podemos llamar el núcleo (formal) de una teoría, simbolizado por K. Se puede decir que $\mathbf{K}$ sintetiza la identidad formal de la teoría. Es formal en el sentido de que todos sus componentes pueden ser definidos, en principio, con toda precisión usando instrumentos formales de la teoría de modelos, la teoría de conjuntos y la topología. Sin embargo, otra tesis básica del estructuralismo es que esta estructura de estructuras no agota todo lo que hay que conocer de una teoría para saber de qué teoría se trata y cómo funciona. La razón de ser de una teoría empírica consiste justamente en el hecho de que todo este aparato formal supuestamente es aplicable a alguna cosa externa a él, a fenómenos que se supone existen independientemente del aparato formal. Este "mundo exterior" es descrito por los estructuralistas, que se apoyan en la noción introducida por Adams, como el "dominio de aplicaciones intencionales", simbolizado por I. Como Adams lo había señalado ya, debe ser considerado asimismo como perteneciente a la identidad de la teoría, puesto que sin él no sabríamos con qué objeto fue construida.

Ahora bien, el estructuralismo hace tres suposiciones epistemológicas fundamentales sobre la manera adecuada de concebir este dominio I. Primeramente, no se trata ciertamente de la "realidad pura" ni de la "experiencia pura" -suponiendo que estas expresiones tengan sentido-. I está conceptualmente determinado por conceptos de los que ya se dispone antes de que la teoría empiece a funcionar. Son conceptos que, con toda seguridad, provienen del "exterior", pero que, en cierto sentido, también pertenecen a la teoría. Para decirlo brevemente, se trata de conceptos T-no-teóricos en el sentido antes explicado. Su combinación coherente forma subestructuras de modelos potenciales de la teoría o, dicho en otros términos, el dominio I debe ser concebido como un subconjunto del conjunto de los modelos potenciales parciales. En segundo lugar, las aplicaciones intencionales de una teoría dada no pretenden referirse a la totalidad del universo o de la experiencia. Son múltiples y locales. Representan "pequeñas partes" de la experiencia humana. Además, cada teoría tiene su 
dominio particular de aplicaciones intencionales, pudiendo coincidir los dominios de teorías diferentes de manera total o parcial, estar en relaciones mutuas o no tener absolutamente ninguna relación entre sí. Finalmente, concibiendo I como un subconjunto del conjunto de modelos potenciales parciales, no damos más que una caracterización bastante débil de este dominio. Se trata solamente de una condición necesaria aunque difícilmente suficiente de pertenencia a I. La determinación unívoca completa de I escapa, por principio, al análisis formal. La razón es que este dominio es una suerte de entidad fuertemente dependiente de factores pragmáticos e históricos que, por su naturaleza misma, no son determinables formalmente. Por consiguiente, en la identidad esencial de toda teoría empírica hay un componente irreductiblemente pragmático-histórico, no formalizable. Se reencuentran aquí los límites del análisis formal de las ciencias empíricas -lo que no quiere decir, obviamente, que no se puedan obtener valiosos resultados en este género de análisis al considerar los aspectos que sí son formalizables-.

En un primer análisis, pues, una teoría es, desde el punto de vista estructuralista, un par $\langle\mathbf{K}, \mathbf{I}\rangle$, donde $\mathrm{K}$ es un núcleo formal e I un dominio de aplicaciones intencionales. Este par está asociado a la "afirmación empírica" según la cual I puede ser efectivamente (o aproximadamente) subsumido bajo K -esto es lo que la teoría "dice acerca del mundo"-. Esta definición de la noción de teoría empírica recuerda, sin duda, la de Adams, para quien una teoría también es un par $\langle\mathbf{M}, \mathbf{I}\rangle$ asociado a una "pretensión empírica"; sin embargo, como podemos ver, el análisis estructuralista se diferencia bastante del de Adams, puesto que, en el caso de los estructuralistas, $\mathbf{M}$ es sólo uno de los componentes del núcleo y la pretensión empírica no postula simplemente que I es un subconjunto de $\mathbf{M}$, sino que existe una relación bastante más compleja entre los dos -la relación de subsunción-.

Acabamos de decir que el estructuralismo concibe una teoría como un par $\langle\mathbf{K}, \mathbf{I}\rangle$ "en un primer análisis". De hecho, el estructuralismo propone concebir las teorías "normales" como estructuras más complejas aún. La razón es que un par del tipo $\langle\mathbf{K}, \mathbf{I}\rangle$ representa sólo el caso más simple que uno puede imaginar de teoría científica -aquél en el que la parte "sustancial" de la teoría consiste sólo en una única ley-. Estas unidades simples son llamadas "elementos teóricos”. No pueden constituir una buena representación de una teoría empírica más que en los casos de disciplinas poco desarrolladas. Pero en el caso de teorías más avanzadas (como lo son prácticamente todas las de las ciencias naturales y la mayor parte de las de las sociales), se puede uno convencer fácilmente de que se trata más bien de "agrupaciones" de un mayor o menor número de elementos teóricos, puesto que contienen varias leyes, de distinto grado de generalidad, subordinadas unas a otras. La denominación específica para estas agrupaciones es la de "red teórica". Estas unidades reflejan el hecho de que las teorías "normales" de las ciencias empíricas tienen la forma de estructuras altamente jerarquizadas ("piramidalmente", si uno considera la imagen gráfica de las mismas). Habitualmente, hay una sola ley fundamental (en general, de contenido esquemático) que constituye el elemento teórico en el vértice más alto de la red y, debajo de él, una serie de leyes (y de condiciones de ligadura) cada vez más especializadas (constituyendo cada una un elemento teórico con su propio dominio de aplicaciones intencionales), que provienen directa o indirectamente de la aplicación de un proceso que podemos llamar de "especificación" (por ejemplo, especificación de las relaciones entre las magnitudes que aparecen en la ley fundamental, concretización de parámetros o de "constantes", restricción de las aproximaciones admitidas, etc.). La reconstrucción estructuralista detallada de docenas de ejemplos de teorías de las disciplinas más variadas ha mostrado que es esta forma de red teórica la que corresponde mejor al concepto intuitivo de teoría que encontramos en los manuales científicos. Para dar un solo ejemplo, que ha sido reconstruido en detalle por los estructuralistas, la teoría a la que habitualmente llamamos "mecánica newtoniana de partículas" consiste en una red en la que el elemento teórico de la cúspide en el vértice más alto- está constituido por el segundo principio de Newton, más un gran número de especializaciones sucesivas, las primeras aún muy generales por sus contenidos y sus dominios de aplicación (tales como el principio de acción y reacción o el postulado según el cual las fuerzas dependen de las distancias), hasta llegar a leyes muy particulares, tales como la ley de Hooke para los 
resortes. ${ }^{6}$ A pesar de esta complejidad, lo que hace que una red pueda ser concebida como una unidad epistemológica y metodológica, es, en primer lugar, el hecho de que dispone de un marco conceptual común (formalmente, que los modelos potenciales sean los mismos) y, en segundo lugar, que todos los elementos teóricos que la componen en sus diversos niveles son siempre construibles como especializaciones (en un sentido que se puede definir formalmente sin ambigüedad) del elemento teórico en la cúspide de la red.

Hasta aquí, hemos visto lo esencial del concepto estructuralista de teoría empírica desde un punto de vista estrictamente sincrónico. Ahora bien, este concepto se puede "poner en marcha" y representar aspectos diacrónicos de las teorías científicas (inspirándose en las ideas de Kuhn de una manera indirecta -aunque aceptada por el propio Kuhn-). En el análisis estructuralista, una teoría en el sentido diacrónico no es simplemente una red teórica que preserva su forma original en el curso de la historia; al contrario, hay que tomar en cuenta el hecho de que las redes teóricas están normalmente sujetas a modificaciones más o menos importantes en el curso de su desarrollo histórico, sin perder sin embargo lo esencial de su identidad. Una teoría, desde el punto de vista diacrónico, es un una red que evoluciona o, para ser más precisos, una secuencia de redes en el tiempo vinculadas por condiciones determinadas. La entidad diacrónica que resulta de este género de proceso ha sido denominada por los estructuralistas "evolución teórica". En cierto sentido, la noción estructuralista de evolución teórica es una precisión (y por ende una mejor base para una contrastación efectiva) de la noción kuhniana de "ciencia normal". También esta noción ha sido aplicada al estudio de casos concretos, tales como la evolución de la mecánica newtoniana y la de la termodinámica fenomenológica.

A pesar de su innegable éxito, una de las críticas que se han hecho al programa estructuralista es que el aparato metateórico empleado es demasiado complicado y que hay que realizar un esfuerzo considerable para "digerirlo" antes de poder aplicarlo al análisis de problemas epistemológicos interesantes. A lo cual los estructuralistas (como yo mismo) replican que es la evolución misma de la filosofía de la ciencia lo que les ha llevado a dar con un nivel de complejidad más elevado, mostrando que los instrumentos conceptuales utilizados por los autores y corrientes precedentes eran demasiado simples y/o demasiado vagos, en todo caso insuficientes, para dar cuenta de ciertos aspectos esenciales de las teorías científicas. Después de todo, las teorías científicas (y sus relaciones) son objetos, ellos mismos, bastante complicados y sería sorprendente que uno pudiera analizar bien objetos tan complejos con instrumentos simples e imprecisos. Sea o no demasiado complicada, la metateoría estructuralista ha mostrado ser aplicable de manera convincente a muchos más casos particulares que las demás metateorías. Es un hecho estadístico fácil de verificar.

Otra crítica frecuente es que aunque la metateoría estructuralista haya sido capaz de reconstruir teorías científicas particulares, no ofrece, sin embargo, ninguna respuesta a los grandes problemas epistemológicos y ontológicos que han preocupado a los filósofos de la ciencia desde los comienzos. El estructuralismo no nos ayuda a decidirnos por concepciones como el empirismo, el realismo, el antirrealismo o el instrumentalismo, ni a determinar el papel de la inducción o la probabilidad en la investigación científica, ni a aclarar las nociones de ley natural o explicación científica... Esta objeción está hasta cierto punto fundada. El silencio del estructuralismo sobre estas cuestiones se debe a razones contingentes (personales), pero también es un resultado de la prudencia: sabemos todavía muy poco acerca de la auténtica naturaleza y el verdadero funcionamiento de las teorías científicas como para estar en condiciones de dar respuestas satisfactorias a todas estas cuestiones. Sin embargo, desde hace algunos años, se han hecho algunos esfuerzos en esta dirección. ${ }^{7}$

\footnotetext{
${ }^{6}$ El lector podrá encontrar la reconstrucción (casi) completa de la red de la mecánica newtoniana, así como la red de otras teorías de la física y la química, en An Architectonic for Science. Otras redes de teorías físicas, biológicas, psicológicas, económicas, etc., han sido reconstruidas en detalle por otros autores.

${ }^{7}$ Ver, por ejemplo, el número especial de la revista Synthese consagrado al estructuralismo (vol. 130, 2002) y compilado por este autor.
} 
Bibliografía

Abreu, C., Lorenzano, P. y C. U. Moulines (2013), "Bibliography of Structuralism III (1995-2012, and Additions)", Metatheoria 3(2): 1-36.

Adams, E. W. (1959), "The Foundations of Rigid Body Mechanics and the Derivation of Its Laws from Those of Particle Mechanics", en Henkin, L., Suppes, P. y A. Tarski (eds.), The Axiomatic Method, Amsterdam: North Holland, pp. 250-265.

Balzer, W., Moulines, C. U. y J. D. Sneed (1987), An Architectonic for Science - The Structuralist Program, Dordrecht: Reidel. (Versión castellana de Pablo Lorenzano: Una arquitectónica para la ciencia. El programa estructuralista, Bernal: Universidad Nacional de Quilmes, 2012.)

Balzer, W. y C. U. Moulines (eds.) (1996), Structuralist Theory of Science. Focal Issues, New Results, Berlin: de Gruyter.

Balzer, W., Moulines, C. U. y J. D. Sneed (eds.) (2000), Structuralist Knowledge Representation: Paradigmatic Examples, Amsterdam: Rodopi.

Bartelborth, Th. (1996), Begründungsstrategien, Berlin: Akademie-Verlag.

Diederich, W., Ibarra, A. y Th. Mormann (1989/1994) "Bibliography of Structuralism", Erkenntnis 30(3): 387-407 y 41(3): 403-418.

Giere, R. (1988), Explaining Science, Chicago: University of Chicago Press. (Versión castellana de Claudia Elisa Gidi Blanchet: La explicación de la ciencia: Un acercamiento cognoscitivo, México: Consejo Nacional de Ciencia y Tecnología, 1992.)

Giere, R. (1992), Cognitive Models of Science, Minneapolis: University of Minnesota Press.

Krantz, D., Luce, R. J., Suppes, P. y A. Tversky (1971-1989), Foundations of Measurement, 3 vols., Nueva York: Academic Press.

Kuhn, T. S. ([1962] 1970), The Structure of Scientific Revolutions, 2a ed., Chicago: University of Chicago Press. (Versión castellana de Carlos Solís: La estructura de las revoluciones cientificas, México: F.C.E., 1986.)

Kuhn, T. S. (1976), "Theory-Change as Structure-Change: Comments on the Sneed Formalism”, Erkenntnis 10(2): 179. 199. (Reimpreso en Kuhn 2000, pp. 176-195. Versión castellana de Antonio Beltrán: "Cambio de teoría como cambio de estructura: comentarios al formalismo de Sneed”, en Kuhn 2000, pp. 211-232.)

Kuhn, T. S. (2000), The Road since Structure: Philosophical Essays 1970-1993, Chicago/London: University of Chicago Press. (Versión castellana de Antonio Beltrán y José Romo: El camino desde la estructura, ensayos filosóficos 1970. 1993, Barcelona/Buenos Aires: Paidós, 2002.)

McKinsey, J. C. C., Sugar, A. y P. Suppes (1953), “Axiomatic Foundations of Classical Particle Mechanics", Journal of Rational Mechanics and Analysis 2: 253-272. (Versión castellana de Adolfo G. de la Sienra: Fundamentos axiomáticos para la mecánica de partículas clásica, Michoacán: Universidad Michoacana de San Nicolás de Hidalgo, 1978.)

Moulines, C. U. (1982), Exploraciones metacientificas, Madrid: Alianza Editorial.

Moulines, C. U. (ed.) (2002), “Structuralism. Special issue”, Synthese 130.

Sneed, J. D. ([1971] 1979), The Logical Structure of Mathematical Physics, 2a ed., Dordrecht: Reidel.

Stegmüller, W. (1973), Theorienstrukturen und Theoriendynamik, Berlin: Springer. (Versión castellana de C. Ulises Moulines: Estructura y dinámica de teorias, Barcelona: Ariel, 1983.)

Stegmüller, W. (1979), The Structuralist View of Theories, Berlin: Springer. (Versión castellana de José Luis Zafio Ferrer: La concepción estructuralista de las teorias, Madrid: Alianza Editorial, 1981.)

Suppe, F. (1967), The Meaning and Use of Models in Mathematics and the Exact Sciences, Tesis doctoral, Ann Arbor: University of Michigan.

Suppe, F. (ed.) (1974), The Structure of Scientific Theories, Urbana, IL: University of Illinois Press. (Versión castellana de Pilar Castrillo y Eloy Rada: La estructura de las teorias cientificas, Madrid: Editora Nacional, 1979.) 
Suppe, F. (1989), The Semantic Conception of Theories and Scientific Realism, Urbana, IL: University of Illinois Press.

Suppes, P. ([1957] 1999), Introduction to Logic, 2a ed., New York: Dover. (Versión castellana de Gabriel Aguirre Carrasco de la 1ª ed. inglesa: Introducción a la lógica simbólica, México: CECSA, 1973.)

Suppes, P. (1988), "Representation Theory and the Analysis of Science", Philosophia Naturalis 25: 254-268.

Suppes, P. (1993), Models and Methods in the Philosophy of Science, Dordrecht: Kluwer.

van Fraassen, B. (1980), The Scientific Image, Oxford: Clarendon Press. (Versión castellana de Sergio Martínez: La imagen científica, México: Paidós/UNAM, 1996.)

van Fraassen, B. (1989), Laws and Symmetry, Oxford: Clarendon Press. 